\title{
Testing the effective scale of Quantum Gravity with the next generation of Gamma Ray Telescopes
}

\author{
O.Blanch, J.Lopez, M.Martinez \\ IFAE, Barcelona, Spain
}

\begin{abstract}
The actual potential of the next generation of Gamma Ray Telescopes in improving the existing tests of an effective Quantum Gravity scale from the study of the propagation delay for gamma rays of different energies coming from a distant astrophysical source is discussed. It is shown that the existence of a cosmological Gamma Ray Horizon, will impose very demanding conditions on the observations of the telescopes to try to test a Quantum Gravity scale close to the Planck mass.
\end{abstract}

Key words: Lorentz Invariance, $\gamma$-rays, Quantum Gravity, Cherenkov Telescopes, Gamma Ray Horizon

PACS: 95.85.Pw, 95.30.Sf

\section{Introduction}

Imaging Cherenkov Telescopes have proven to be the most successful tool developed so far to explore the cosmic gamma rays of energies above few hundred $\mathrm{GeV}$. A pioneering generation of installations has been able to detect a handful of sources and start a whole program of very exciting physics studies. Now a second generation of more elaborated telescopes is under construction and will provide soon with new observations. One of the main characteristics of these new telescopes is the ability to detect lower energy gamma rays, which could fill the observational gap between 10 and $300 \mathrm{GeV}$.

One of the most interesting results in fundamental physics from the existing telescopes is a limit, given by the Whipple collaboration [1], on the Quantum

Email addresses: blanch@ifae.es (O.Blanch), jlopez@ifae.es (J.Lopez), martinez@ifae.es (M.Martinez).

Preprint submitted to Elsevier Science 8 July 2021 
Gravity scale to be larger than $4 \times 10^{16} \mathrm{GeV}$ (about $1 / 250$ th of the Planck mass) at the $95 \%$ confidence level. In that study, the data from a TeV gammaray flare associated with the active galaxy Markarian 421 observed on 15 May 1996 was used to place bounds on the possible energy-dependence of the speed of light in terms of an effective scale for quantum gravitational effects.

The basic idea behind the measurement is that gamma rays traversing cosmological distances should notice the quantum fluctuations in the gravitational vacuum which unavoidably should happen in any quantum theory of gravitation. These fluctuations may occur on scale sizes as small as the Planck length $L_{P} \simeq 10^{-33} \mathrm{~cm}$ or time-scales of the order of $t_{P} \simeq 1 / E_{P}\left(E_{P} \simeq 10^{19} \mathrm{GeV}\right)$.

These gammas will therefore experience a "vacuum polarization" correction which should be very small $\left(O\left(E / E_{Q G}\right)\right.$ where $E$ is the gamma energy and $E_{Q G}$ is an effective scale for Quantum Gravity, which might be as large as $E_{P}$ ) but might become measurable after the gamma has traversed cosmological distances. In this Quantum Gravity scenario, the requirement of violation of the Lorenz-Invariance symmetry [3,4] emerges naturally, providing an energydependent propagation speed for electromagnetic waves. Therefore, gammas of different energies being emitted simultaneously by a distant source should reach our observatories at different times.

In fact, for practical purposes, this delay with respect to the ordinary case of an energy-independent speed c for massless particles, for a source at a distance L can be expressed as [4]

$$
\Delta t \sim \xi \frac{E}{E_{Q G}} \frac{L}{c}
$$

This delay should be one of the cleanest signatures that Cherenkov telescopes are able to study in order to spot Quantum Gravity effects. Nevertheless, as it has already been pointed out in the literature, it might be mimicked by effects related to the source physics (internal production delays for higher energy gammas) or propagation (time delay due to cascading in intergalactic magnetic fields [5]). Therefore it will be mandatory to use the scaling of the effect with redshift to distinguish between Quantum Gravity effects and any source-dependent phenomena [1].

The new generation of telescopes, should have an improved sensitivity and lower threshold. The first characteristic might help in resolving faster time structures while the second one should allow to observe sources at much longer distances because of a smaller absorption in the intergalactic medium. Therefore, some clear improvement in this kind of measurements should be expected.

The goal of this work is trying to estimate how these characteristics would 
actually help in improving the quest for Quantum Gravity effects. For that, this writeup is organized as follows: first, a very brief overview of the theoretical framework used in this study is presented. Second, the results of the expected delay as a function of the gamma ray energy and the source redshift location for different Quantum Gravity scenarios is discussed. Third, the effect

of the existence of a Gamma Ray Horizon, including consistently the effect of the Lorenz Invariance Violation, is shown. After that, a discussion about the observational picture when all these effects are put together is presented and finally, in the conclusions we give our prospects for these observations with the coming generation of Cherenkov telescopes.

\section{Overview of the theoretical framework}

When developing any model for Quantum Gravity, it appears naturally the necessity to modify some of the most basic continuous symmetries of spacetime, such as Lorentz Invariance $[2,3,4,6]$. In this analysis we shall focus in the violation of the Lorentz Invariance symmetry (LIV) caused by Quantum Gravity because it is the responsible of the main changes in the kinematics in which is based this study.

The violation of the Lorentz Invariance symmetry (LIV) modifies the dispersion relation giving the propagation speed for gamma rays in a theorydependent manner. However, since in all plausible approaches the actual effects are expected to be small, they can be studied from a phenomenological point of view using an expansion in terms of the gamma energy divided by the effective Quantum Gravity scale. Therefore, the actual gamma dispersion relation for a massless particle can be expressed in leading order as [7]

$$
E^{2}-c^{2} \vec{p}^{2} \simeq E^{2} \xi\left(\frac{E}{E_{Q G}}\right)^{\alpha}
$$

where $E$ and $\vec{p}$ denotes the energy and the momentum of gammas, $\xi$ and $\alpha$ are the LIV free parameters and $E_{Q G}$ is the energy scale for Quantum Gravity.

In this scenario, gamma rays travelling in vacuum can be seen like traversing a material medium [9]. Hence, the propagation speed of gamma rays should be computed as

$$
v=\frac{d E}{d p}=c\left[1+\xi \frac{1+\alpha}{2}\left(\frac{E}{E_{Q G}}\right)^{\alpha}\right]
$$

where $E$ is actually the gamma comoving energy. In terms of the measured 
gamma energy at the Earth $E_{\gamma}$, the velocity when the gamma is at a redshift $z$ is

$$
v=\frac{d E}{d p}=c\left[1+\xi \frac{1+\alpha}{2}\left(\frac{E_{\gamma}(1+z)}{E_{Q G}}\right)^{\alpha}\right]
$$

Therefore, the actual time-of-flight for a gamma ray coming from a source at redshift $z$, is given by

$$
t=\int_{0}^{z} \frac{c}{v} \frac{d t}{d z} d z
$$

where

$$
\frac{d t}{d z}=\frac{1 /(1+z)}{H_{0}\left[\Omega_{M}(1+z)^{3}+\Omega_{K}(1+z)^{2}+\Omega_{\Lambda}\right]^{1 / 2}}
$$

being $H_{0}$ the Hubble constant and $\Omega_{M}, \Omega_{K}$ and $\Omega_{\Lambda}{ }^{1}$ the standard cosmological parameters.

For practical purposes, we need the difference in the time-of-flight for gamma rays of different energies $\left(E_{\gamma}\right.$ and $\left.E_{\gamma}^{\prime}\right)$ produced at the same redshift $z$ because this is the signature that Gamma Ray Telescopes are able to study. This difference is given by

$$
\begin{aligned}
\Delta t=t_{E_{\gamma}}-t_{E_{\gamma}^{\prime}} & =\int_{0}^{z}\left(\frac{c}{v_{E_{\gamma}}}-\frac{c}{v_{E_{\gamma}^{\prime}}}\right) \frac{d t}{d z} d z \\
& \simeq-\xi \frac{1+\alpha}{2} \frac{E_{\gamma}^{\alpha}-E_{\gamma}^{\alpha}}{E_{Q G}^{\alpha}} \int_{0}^{z}(1+z)^{\alpha} \frac{d t}{d z} d z
\end{aligned}
$$

If in the above expressions we take $\alpha=1, E_{\gamma}>>E_{\gamma}^{\prime}$ and the limit $z<<1$, we recover the simple expression used by the Whipple collaboration.

$$
\Delta t \sim \xi \frac{E}{E_{Q G}} \frac{z}{H_{0}}=\xi \frac{E}{E_{Q G}} \frac{L}{c}
$$

$\overline{1}$ In this analysis we have used the current best fit values for the fundamental cosmological parameters $H_{0}=68 \pm 6 \mathrm{~km} \mathrm{~s}^{-1} \mathrm{Mpc}^{-1}, \Omega_{M}=0.35 \pm 0.1$ and $\Omega_{\Lambda}=0.65 \pm 0.15$ following reference [8]. 


\section{The expected delay}

The free parameters used in our phenomenological approach are the ones used in references [7], namely $\xi, \alpha$, and $E_{Q G}$. $\xi$ is only a sign ambiguity and hence its possible values are $\xi= \pm 1$. Out of the two possible values, in this work we have chosen to use $\xi=-1$ because it fits better the present experimental constraints $[6,10]$. In what concerns $\alpha$, in Quantum Gravity theories corrections going like $\left(E / E_{p}\right)^{\alpha}$, where $E_{p}$ is the Planck energy, typically appear as leading order of more complex analytic expressions $[7,10]$. This motivates us to study the cases $\alpha=1$ and 2 . Nevertheless, $\alpha=2$ means an $E_{Q G}$ suppression factor in the difference of time of flight. This would lead to a non measurable $\Delta t$ $\left(<10^{-15} s\right)$. Therefore we cannot exclude the possibility that the leading term of LIV goes as $\left(E / E_{Q G}\right)^{2}$ but, if it was the case, the Gamma Ray Telescopes would not have any chance to test it with the gamma time-of-flights. That forces us to study only the most favourable case (potentially largest Quantum Gravity effects) hence $\alpha=1$.

In this scenario equation 7 is reduced to

$$
\Delta t \sim \frac{\Delta E}{E_{Q G}} \int_{0}^{z}(1+z) \frac{d t}{d z} d z
$$

Figure 1 shows the lines in the plane $\Delta E$ versus $\Delta t$ provided by the above equations for a set of different source redshifts. From this plot it is clear that, for a given energy difference between the detected gammas, being capable of observing sources at larger redshifts should allow to check the $E_{Q G}$ with less demanding time resolutions. Or conversely it should allow, for a fixed time resolution, to use lower energy gammas to check the $E_{Q G}$. This should help because, given the steep power-law energy spectrum of all the known high energy gamma astrophysical sources, lowering the gamma energy scale should allow to observe a larger number of gammas and, hence, to improve the experimental accuracy.

Nevertheless, in spite of the fact that the effective distance integral giving the $z$ dependence of the time delay has a correction of $(1+z)$ to the lookback time integrand (which increases the effective gamma propagation time) it is clear from figure 1 that this dependence on the redshift saturates quite quickly.

Actually the main target of the time-of-flight studies is to explore the $E_{Q G}$ scale. In figure 1 it is shown the effect of observing sources at different redshifts to test a given value of $E_{Q G}\left(E_{Q G}=E_{p}\right)$. Alternatively, fixing the observable delay time scale, this result can be shown as in figure 2, as the capability of exploring higher Quantum Gravity scales as the source redshift increases. 


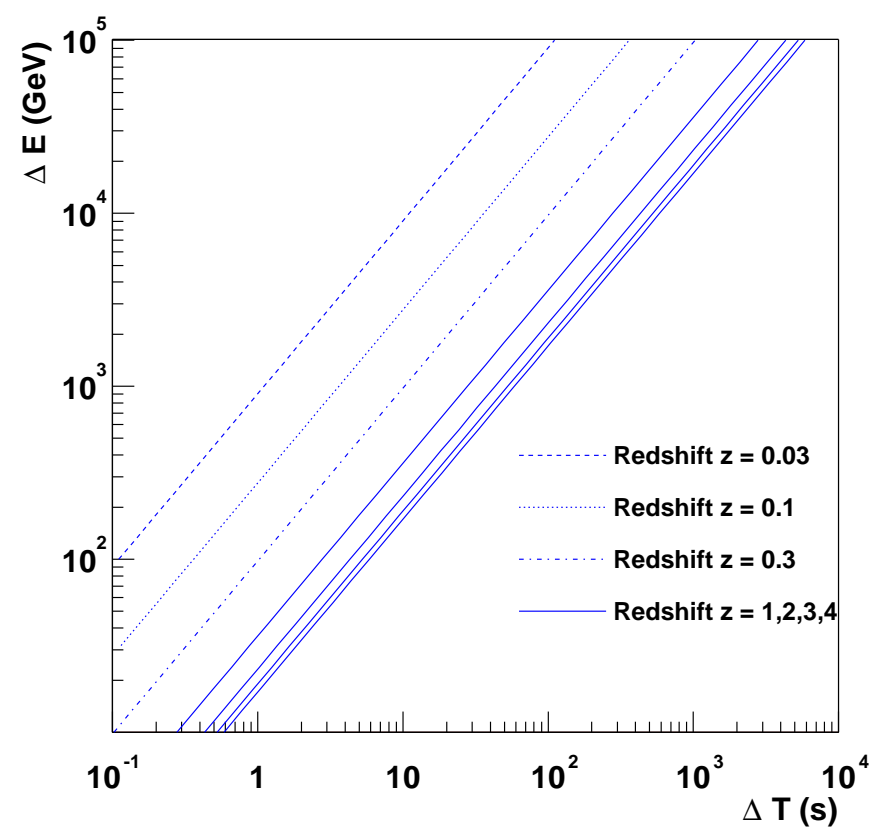

Fig. 1. Expected delay as a function of the gamma ray energy for different redshift sources and a value of $E_{Q G}=E_{p}$.

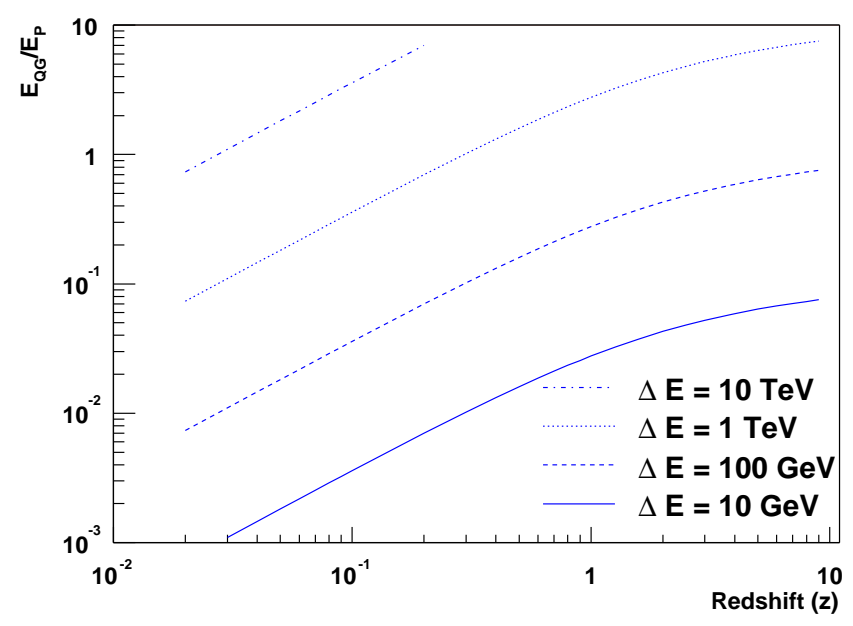

Fig. 2. $E_{Q G} / E_{p}$ versus the redshift $z$ of the gamma ray source for different $\Delta E$ values and fixed $\Delta t=10 \mathrm{~s}$.

\section{The modified Gamma Ray Horizon}

The above considerations about the benefit of observing higher redshift sources to explore higher Quantum Gravity effective scales were implicitly assuming that the gamma rays traverse the intergalactic space without being altered. In reality, we expect high energy gamma rays traversing cosmological distances to be absorbed by their interaction with the diffuse background radiation fields, or "Extragalactic Background Light" (EBL), producing $e^{+} e^{-}$pairs. 


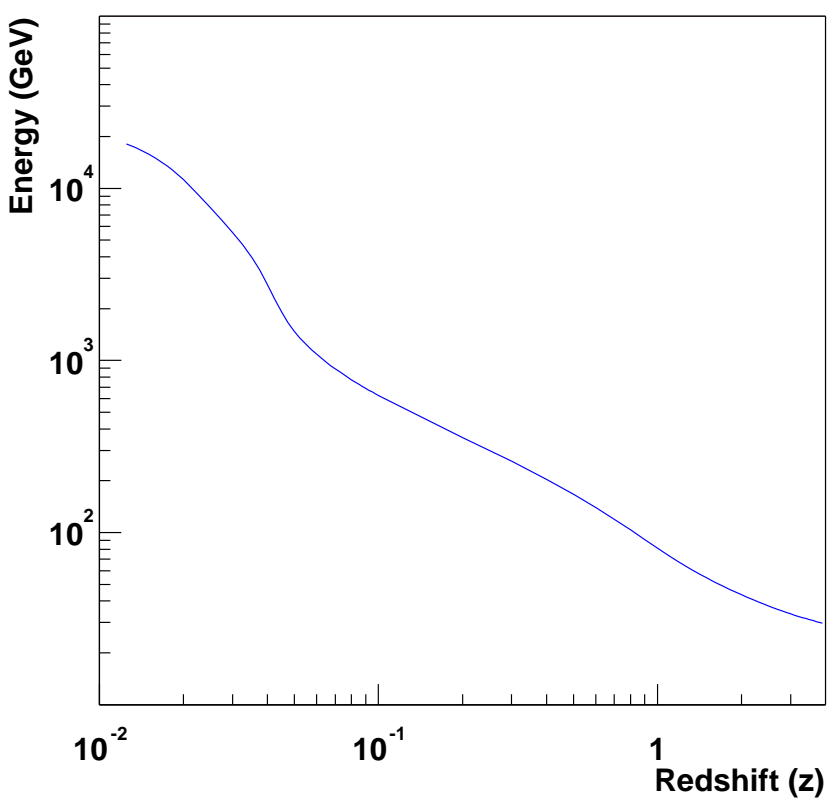

Fig. 3. The Gamma Ray Horizon computed assuming purely Standard Model Physics and using the EBL model presented in the reference [14].

The $\gamma_{H E} \gamma_{E B L} \rightarrow e^{+} e^{-}$cross section is strongly picked to $E_{C M} \sim 1.8 \times\left(2 m_{e}\right)$ and therefore, there is a specific range in the EBL energy which is "probed" by each gamma ray energy [11].

This effect should lead to the existence of a "Gamma Ray Horizon", limiting the feasibility of observing very high energy gamma rays coming from very far distances. The actual value of this horizon distance for gamma rays of a given energy, depends on the number density of the diffuse background radiation of the relevant energy range, which is traversed by the gamma rays. In the range of gamma ray energies which can be effectively studied by the next generation of Gamma Ray Telescopes (from, say, $10 \mathrm{GeV}$ to $50 \mathrm{TeV}$ ), the most relevant EBL component is the infrared contribution.

Quite precise predictions ${ }^{2}$ of the Gamma Ray Horizon have been made but, unfortunately, so far no clear confirmation can be drawn from the observations of the present generation of Gamma Ray Telescopes. In this work we have used the approach and procedures described in $[11,12,13]$ which predict a Gamma Ray Horizon as shown in figure 3.

Nevertheless, the above prediction has been obtained by assuming purely Stan-

$\overline{2}$ In spite of the precision of these predictions they have still rather considerable systematic uncertainties due to the poor knowledge of some of their ingredients (direct measurement of the EBL at small redshifts and theoretical extrapolation to large redshifts) and are therefore quite inaccurate. This situation is, nevertheless, improving quite quickly with a whole harvest of new measurements. 
dard Model Physics. Recent claims of observation of very high energy gamma ray events coming from Mkn 501 [15], a blazar at redshift $z \sim 0.03$ which would somehow contradict the above prediction of the Gamma Ray Horizon, have motivated some authors $[10,16,17,18]$ to revise that prediction. It has been pointed out that the actual existence of LIV should also affect the calculation of the Gamma Ray Horizon, since the threshold energy of the EBL needed to produce $e^{+} e^{-}$pairs is modified [10]. This effect could explain the very high energy observed events.

Therefore, to be consistent with the framework of our analysis, we've also undertaken the task of implementing consistently in the complete calculation of the Gamma Ray Horizon the inclusion of the threshold-modifying effects coming from the effective Quantum Gravity inspired dispersion relations discussed in the previous sections. For that we've followed the approach of reference [10], where the modified threshold value for the gamma ray momentum to produce $e^{+} e^{-}$pairs is deduced ${ }^{3}$

$$
p_{t h}=\frac{m_{e}^{2}}{\epsilon}+\xi \frac{p_{t h}^{2+\alpha}}{4 \epsilon E_{Q G}^{\alpha}}\left(\frac{1}{2^{\alpha}}-1\right)
$$

where $\epsilon$ is the energy of the EBL gammas and $p_{t h}$ it the threshold momentum for the gamma ray.

In the standard calculation of the Gamma Ray Horizon [19], the optical depth for gamma rays of energy $E_{\gamma}$ coming from a source at redshift $z$ is obtained from the integration

$$
\tau\left(E_{\gamma}, z\right)=\int_{0}^{z} d z^{\prime} \frac{d l}{d z^{\prime}} \int_{0}^{2} d x \frac{x}{2} \int_{\epsilon t r}^{\infty} d \epsilon \cdot n\left(\epsilon, z^{\prime}\right) \sigma\left[2 x E_{\gamma} \epsilon\left(1+z^{\prime}\right)^{2}\right]
$$

where $x \equiv 1-\cos \theta$ being $\theta$ the gamma-gamma scattering angle, $n\left(\epsilon, z^{\prime}\right)$ the spectral density of the EBL gammas $n(\epsilon)$ at the given z' and $\epsilon_{t h}$ the threshold energy for the EBL gammas which is given by

$$
\epsilon_{t r}=\frac{2 m_{e}^{2}}{E_{\gamma} x\left(1+z^{\prime}\right)^{2}}
$$

After we include LIV, we arrive to a modified threshold condition of

$$
\epsilon_{t r}=\frac{2 m_{e}^{2}}{E_{\gamma} x\left(1+z^{\prime}\right)^{2}}+\xi \frac{2}{x\left(1+z^{\prime}\right)} \frac{\left[E_{\gamma}\left(1+z^{\prime}\right)\right]^{1+\alpha}}{4 E_{Q G}^{\alpha}}\left(\frac{1}{2^{\alpha}}-1\right)
$$

$\overline{3}$ In [10], the notation is slightly different. There appears a parameter $\eta$, which is $\xi \cdot\left(E_{p} / E_{Q G}\right)^{\alpha}$ 


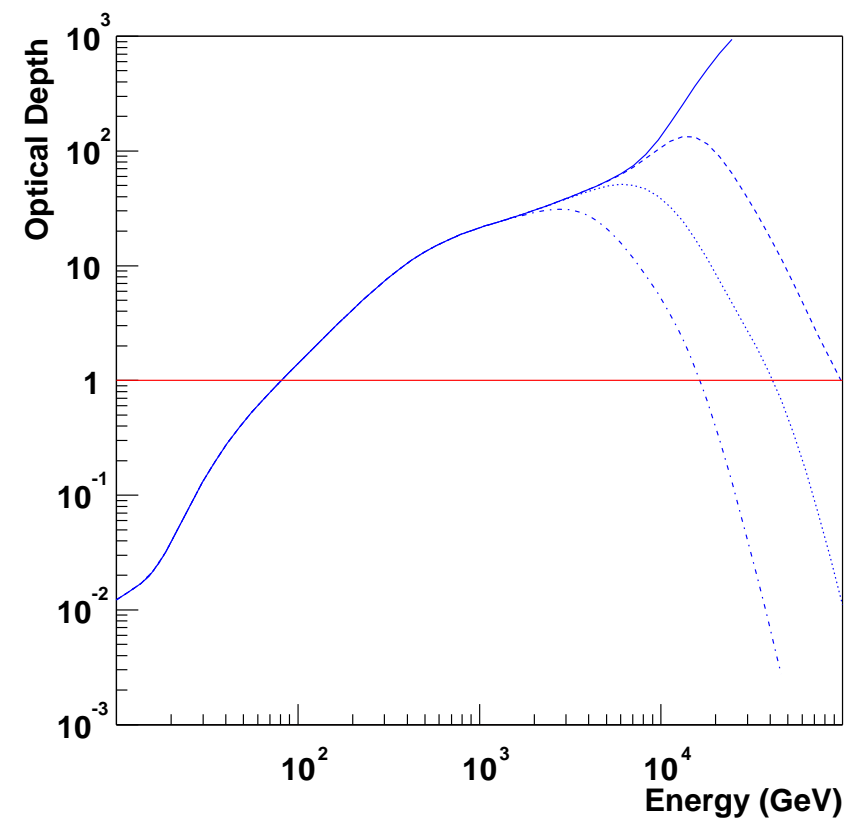

Fig. 4. The Optical Depth as a function of the gamma ray energy for a redshift $z=1$.The continuous line is for a calculation without LIV whereas the dashed, dotted and dash-dotted lines are for values of $E_{Q G}=E_{p}, E_{p} / 10$ and $E_{p} / 100$ respectively. The horizontal line shows that the Gamma Ray Horizon is defined for the energies were the Optical Depth is equal to one.

where $E_{\gamma}$ and $\epsilon_{t r}$ are the energies at the Earth and, therefore, we had to add some $\left(1+z^{\prime}\right)$ factors respect to the equation 10 . The dependence on the scattering angle has also been introduced as a global factor $2 / x$.

The results comparing the optical depths with and without this threshold correction (once again in the $\alpha=1$ and $\xi=-1$ scenario) are shown in figure 4 . For these parameter values the net effect of the correction is increasing the threshold energy in such a way that, for any given gamma energy, harder EBL photons are responsible for their absorption.

In the Optical Depth calculation without LIV, the gamma rays of $10^{5} \mathrm{GeV}$ "probe" the EBL spectrum at around $\lambda_{E B L} \sim 100 \mu m$ where the density has a maximum, while when the LIV correction is present, they probe the EBL spectrum at lower wavelengths, where the value of the density is smaller [12]. The main consequence of this is that in the calculation with LIV corrections the universe becomes transparent again for large energy gammas whereas the results for the lower energy gammas of the plots remain basically unchanged. The correction for large energy gammas is so severe that avoids the existence of a Gamma Ray Horizon for moderate redshift sources, like Mkn 421, giving then a plausible explanation for the observed gamma events as has already being mentioned. 


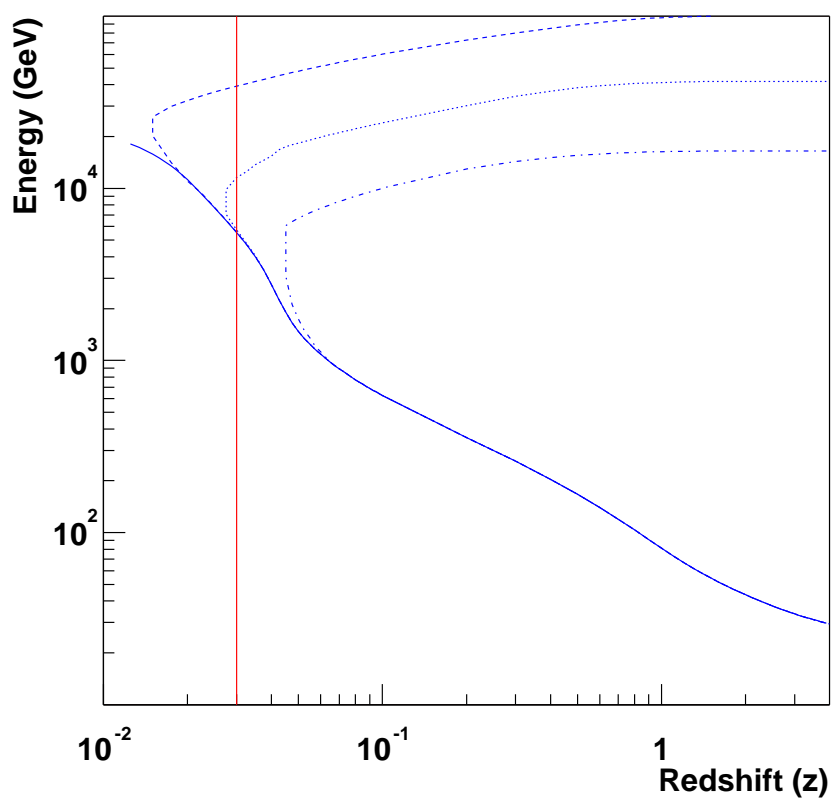

Fig. 5. The Gamma Ray Horizon computed assuming LIV. The continuous line is for a calculation without LIV whereas the dashed, dotted and dash-dotted lines are for values of $E_{Q G}=E_{p}, E_{p} / 10$ and $E_{p} / 100$ respectively. Notice that for $E_{Q G} \sim$ $E_{p} / 10$ the Gamma Ray Horizon disappears for the redshift of the observed blazars Markarian 421 and $501(z \sim 0.03)$, which is shown in the plot with a vertical line.

The obtained Gamma Ray Horizon is shown in figure 5 for different $E_{Q G}$ and compared with the "standard" one. If one assumes high $E_{Q G}$, it is clear from that plot that the main difference due to LIV should happen for low and moderate redshift sources whereas for large redshift sources no effect should be observable in the Gamma Ray Horizon. On the other hand for not so high $E_{Q G}$, the universe becomes transparent again for a gamma ray energy that can be reached with current AGN models [20] (15 TeV for $\left.E_{Q G}=E_{p} / 100\right)$. The fact that $\gamma$ rays of this high energies have been detected for close objects $(z=0.03)[15]$ but not for objects at larger redshift suggests that the $E_{Q G}$ must be in excess of $\approx E_{p} / 100$. Actually, one of the most recent results about the effects of LIV in the Gamma Ray Horizon was presented in [21] where $\mathrm{TeV}$ gamma ray observations of the active galaxy Markarian 501 were used to set a lower limit on the Lorentz Invariance breaking parameters.

\section{Discussion}

We shall now put together the effects discussed in the previous sections to get a complete picture of the expectations. As already mentioned, on the one hand the capability of observing more distant sources should allow to have more lever arm to explore higher effective Quantum Gravity scales. Unfortunately, 
on the other hand, for more distant sources, the gamma absorption in the EBL is stronger and the Gamma Ray Horizon happens at smaller gamma energies.

This trade-off is clearly summarized in figures 6 where, on top of the $\Delta E$ versus $\Delta t$ lines predicting the propagation delays, one can see the parameter region beyond the Gamma Ray Horizon. It must be stressed here that the Gamma Ray Horizon does not mean a "hard boundary" since it just gives the condition for an e-fold reduction of the observed flux. Because of that, the shaded area given by the Gamma Ray Horizon has to be understood as the region in which the flux reduction due to the absorption starts being strong enough to make the source observation difficult.

From these figures it is clear that the time resolution needed depends strongly on the actual effective Quantum Gravity scale that one would like to test. To explore up to the Planck mass, time resolutions on the scale of just few seconds will be mandatory if gamma rays of up to few hundred $\mathrm{GeV}$ are used (or few tens of seconds if gammas of few $\mathrm{TeVs}$ are used) quite independently on the actual source redshift. But resolution of several minutes could be enough for a scale of one hundredth of the Planck mass.

In view of these figures it is not obvious how much having a reduced threshold would help the next Cherenkov Telescope generation to improve in this kind of analysis. In fact, from the above figures the straight conclusion is that the observation of the nearby Blazars (Mkn 421, at $z=0.031$ is actually the closest known Blazar) in an energy range of $10^{3}-10^{4} \mathrm{GeV}$ seems to be the most comfortable scenario for exploring the highest effective Quantum Gravity scale.

\section{Conclusions}

In this work the potential of the next generation of Gamma Ray Telescopes in improving the existing tests of an effective Quantum Gravity scale from the study of the propagation delay for gamma rays of different energies coming from a distant astrophysical source has been analyzed. These new telescopes should have better sensitivity and lower threshold than the present ones, enabling a better time resolution and the observation of sources at higher redshift and therefore, a clear improvement in this kind of measurements should be expected.

Detailed calculations on the actual expected delay for sources at any redshift have been presented. They show that the effective delay time grows with the redshift faster than the lookback time. In spite of that, the redshift dependence of the relationship between the gamma energy scale and the observable arrival 

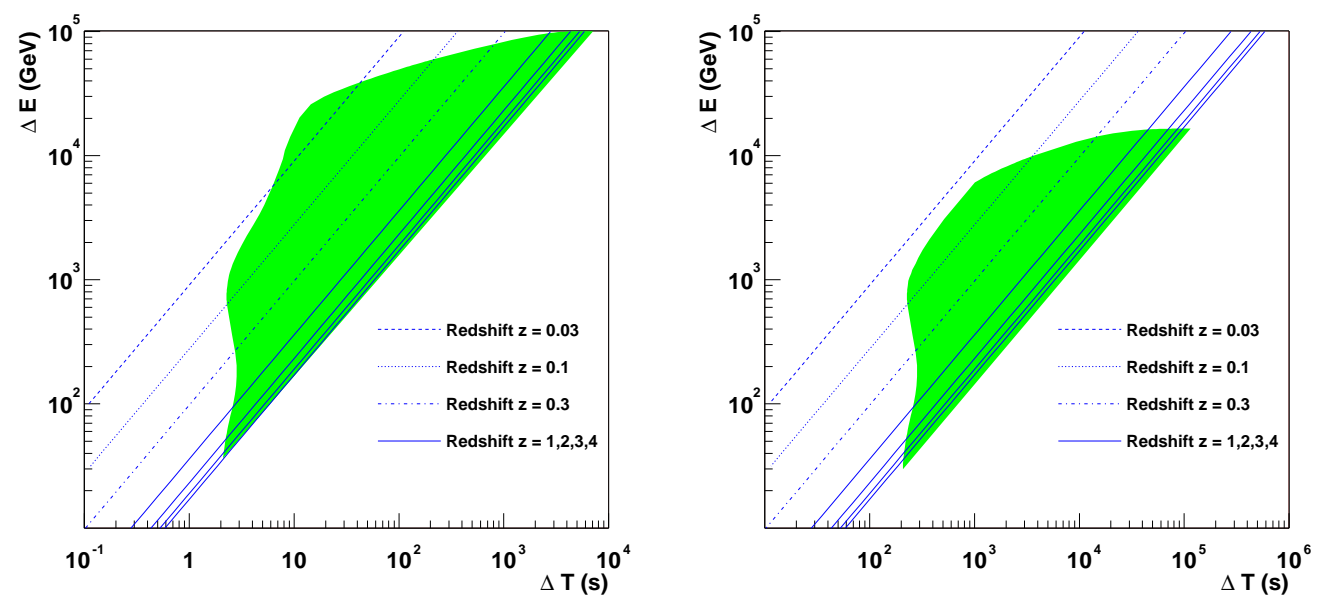

Fig. 6. Expected delay as a function of the gamma ray energy for different redshift sources. The shadow area is a projection of the Gamma Ray Horizon in the $\Delta E$ v.s. $\Delta t$ plane. Therefore, the possible Quantum Gravity effects can hardly be studied in this area due to the lack of gamma rays. $E_{Q G}=E_{p}$ and $E_{p} / 100$ for the left and right plots respectively. Notice the change in the time scale axis for the different plots.

time delay has been shown to saturate rather quickly as the source redshift increases.

In addition, a calculation of the Gamma Ray Horizon due to the gamma absorption in the extragalactic background light (EBL) taking consistently into account the Lorenz Invariance Violation (LIV) effects has been carried out. This absorption should limit the observability of high energy gammas coming from very distant sources but, as it has already been realized in the past, the inclusion of LIV effects opens a window for very high energy gammas.

Putting all these effects together, the global picture is that quite stringent requirements on the time resolution (in the scale of few seconds) should be achieved to be able to explore an effective Quantum Gravity scale close to the Planck mass. In fact, given the effect of the Gamma Ray Horizon, this demanding time resolution constraint cannot be avoided by using large redshift sources unless very high energy gamma rays are used. Although, this resolution is less restrictive (several minutes) for a scale around one hundredth of the Planck mass. In view of this situation, maybe the observation of the nearby Blazars in an energy range of $10^{3}-10^{4} \mathrm{GeV}$ could be the most comfortable scenario for exploring the highest effective Quantum Gravity scale. Given this result, the new generation of telescope arrays might be the most promising choice since they can provide a much larger collection area for higher energy gamma rays. 


\section{Acknowledgements}

We would like to thank our colleagues of the MAGIC collaboration for their comments and support. We are indebted to S.M.Bradbury from the VERITAS collaboration for triggering our interest in this subject. We thank R.Pascual for discussions and comments on this manuscript. We'd like also to thank J.Cortina, O. de Jagger, J.Garriga, A.Grifols, E.Lorenz, K.Mannheim and A.Saggion for their encouraging comments. And finally, we'd like to thank to O. Bertolami, G. Amelino-Camelia and F. Stecker for their comments in the preprint stage of this article.

\section{References}

[1] S.D. Biller et all, Phys. Rev. Lett. 83 (1999) 2108.

[2] L. Gonzalez-Mestres, physics/9704017.

[3] S. Coleman and S.L. Glashow, Phys Rev. D59 (1999) 116008.

[4] G. Amelino-Camelia, J. Ellis, N.E. Mavromatos, D.V. Nanopoulos and S. Sarkar, Nature 393 (1998) 763.

[5] R. Plaga, Nature 430 (1995) 374.

[6] G. Amelino-Camelia and T. Piran, Phys Lett. B497 (2001) 265.

[7] G. Amelino-Camelia, notes based on lectures given at the XXXV Karpacz Winter School of Theoretical Physics From Cosmology to Quantum Gravity, Polanica, Poland, 2-12 February, 1999

[8] L.M. Krauss, submitted to World Scientific on 27 Mar 2001.

[9] J.I. Latorre, P. Pascual and R. Tarrach, Nucl. Phys. B437, (1995) 60.

[10] G. Amelino-Camelia and T. Piran, Phys. Rev. D64 (2001) 036005.

[11] V. Vassiliev, Astropart. Phys. 12 (2000) 217 and references therein.

[12] O. Blanch, M. Martinez, astro-ph/0107582

[13] T.M. Kneiske and K. Mannheim, to appear in Proc. of 33rd ESLAB Symp. (ESA SP-445, 2000).

[14] T.M. Kneiske et al, Astronomy \& Astrophysics 386, p.1 (2002)

[15] R.J. Protheroe et al, based on several talks given at the International Cosmic Ray Conference (ICRC 97), Durban, South Africa, 28 Jul-8 Aug 1997.

[16] H. Sato, Proceedings of the International Space Factory on JEM/ISS workshop, Tsukupa, Japan, 7-9 June 1999. 
[17] R. Aloisio et al, Phy. Rev. D62 (2000) 053010.

[18] T. Kifune, Astrophys. J. 518 (1999) L21.

[19] F.W. Stecker and O.C. De Jager, Absorption of High Energy Gamma-Rays by Low Energy Intergalactic Photons, astro-ph/9501065, Space Sci.Rev. 75 (1996) 401-412.

[20] K. Mannheim, Review talk given at the XXXII Recontres de Moriond, "Very High Energy Phenomena in the Universe", Les Arcs, France, 1997.

[21] F.W. Stecker and S.L. Glasow, Astropart. Phys. 16 (2001) 97 